\title{
Alexander Kästner, Tödliche Geschichte(n). Selbsttötungen in Kursachsen im Spannungsfeld von Normen und Praktiken (1547-1815)
}

Konstanz : UVK (Konflikte und Kultur - Historische Perspektiven, 24), 2012, 676 p., 69,00€

\section{Falk Bretschneider}

\section{OpenEdition}

\section{Journals}

Édition électronique

URL : http://journals.openedition.org/ifha/7792

DOI : 10.4000/ifha.7792

ISSN : 2198-8943

Éditeur

IFRA - Institut franco-allemand (sciences historiques et sociales)

Référence électronique

Falk Bretschneider, «Alexander Kästner, Tödliche Geschichte(n). Selbsttötungen in Kursachsen im Spannungsfeld von Normen und Praktiken (1547-1815) », Revue de l'IFHA [En ligne], Date de recension, mis en ligne le 04 mars 2014, consulté le 22 septembre 2020. URL : http://journals.openedition.org/ ifha/7792 ; DOI : https://doi.org/10.4000/ifha.7792

Ce document a été généré automatiquement le 22 septembre 2020.

(CIFHA 


\section{Alexander Kästner, Tödliche Geschichte(n). Selbsttötungen in Kursachsen im Spannungsfeld von Normen und Praktiken (1547-1815)}

Konstanz : UVK (Konflikte und Kultur - Historische Perspektiven, 24), 2012,676 p., $69,00 €$

Falk Bretschneider

Comme l'indique le titre de cet ouvrage issu d'une thèse de doctorat soutenue en 2010 à l'université de Dresde, A.K. reprend la question théorique désormais ancienne du rapport entre normes et pratiques pour l'appliquer à un thème qui, lui non plus, n'est pas en soi un nouvel objet de recherche des historiens (rappelons les travaux de Vera Lind - voir Bulletin de la MHFA, 36, 2000, p. 277-278 - restés malheureusement quelque peu dans l'ombre), mais qui se trouve traité ici avec une rigueur méthodologique et une diligence empirique exemplaires. En s'intéressant à l'histoire du suicide en Saxe lors de cette longue période qui va de la naissance, en 1547, de la principauté électorale saxonne des Albertins à la défaite, en 1815, du royaume de Saxe allié à Napoléon et à la cession de larges pans du territoire à la Prusse triomphante, l'auteur s'attelle effectivement à une tâche gigantesque : saisir - autant dans les discours théologiques et juridiques que dans les pratiques administratives et sociales, et ceci sur près de trois siècles - les manières d'appréhender ce phénomène bouleversant et tragique qu'était et que demeure le fait de mettre fin à sa propre vie. La structure de l'ouvrage est assez originale : les trois grandes parties de l'analyse ne suivent que partiellement un ordre chronologique, après une introduction qui évalue l'état des recherches et présente les sources dépouillées, mais qui élabore également un cadre conceptuel inscrit dans la lignée des travaux sur l'histoire de la criminalité, de la police et de la domination à l'époque moderne et prenant donc pour horizon la notion d'«acclimatation" des normes (Normimplementierung). 
La première partie pose d'abord la question plus générale du " pourquoi », c'est-à-dire celle des possibilités dont dispose l'historien pour reconstruire les mobiles ayant conduit tel ou tel malheureux au suicide. La réponse que donne A.K. renvoie aux ambiguïtés des sources qu'il a exploitées et donc aussi aux différents contextes dans lesquels celles-ci ont été produites (témoignages de proches ou rapports sommaires d'officiers locaux, attestations de pasteurs ou expertises de médecins, décisions de tribunaux ou d'administrations, etc.). Autrement dit: il n'y a pas de réponses claires, mais un espace d'interprétations possibles, étroitement liées aux cadres matériels et juridiques des enquêtes entreprises par les autorités après chaque cas de suicide et $\mathrm{au}(\mathrm{x})$ sens que donnaient à cet événement les multiples acteurs impliqués. Perçu avant tout comme un crime, le suicide provoquait d'ordinaire les mêmes réactions que n'importe quelle autre infraction majeure, et les sanctions qui en découlaient s'inscrivaient dans la même hétérogénéité et dans la même flexibilité des pratiques pénales modernes, telles que l'historiographie les a détaillées ces dernières années (p.58). Le point névralgique de toute procédure administrative était la décision concernant les modalités d'enterrement (honorable ou non, selon l'état mental et la conduite - Lebenswandel - du suicidé avant sa mort; d'autres sanctions, comme la confiscation des biens, l'exécution d'une peine capitale post mortem ou l'autodafé de la dépouille ne semblent pas avoir été pratiquées en Saxe, p. 222). Ces modalités ont été négociées entre une multitude d'acteurs institutionnels et individuels et notamment entre les autorités laïques et ecclésiastiques (en Saxe donc surtout les tribunaux et les consistoires) auxquelles pouvaient se joindre, par le biais des suppliques, les parents ou amis de la victime. De ce jeu social complexe, l'historien ne peut souvent qu'extraire les "vérités en concurrence " des contemporains (pour reprendre une expression forgée par Andrea Griesebner, citée p. 83 sq.), situation que ne peuvent guère débroussailler les explications scientifiques actuelles (comme celles que proposent par exemple la psychologie ou la neuropathologie).

Les bases d'un traitement normatif du suicide (celles qui existaient avant que le suicide ne commence à être pris en compte par la législation, autour de 1700) font l'objet de la deuxième partie. Ici, l'auteur étudie d'abord les préceptes des théologiens, en premier lieu ceux de Luther (qui, contrairement à ce que la recherche a longtemps affirmé, a adopté des positions contradictoires) et de Melanchthon, tous les deux décisifs dans ce berceau de la Réforme que fut la Saxe. En associant cette analyse à celle d'une source nouvelle - les "collections de conseils» (Conciliensammlungen) qui rassemblent des expertises émises par des instances théologiques ou des savants - et à quelques études de cas, A.K. décortique l'appréciation nuancée que la doctrine luthérienne porte sur le suicide. Pour elle, seules les circonstances concrètes de chaque acte individuel permettaient d'arriver à un jugement moral définitif. Peu présent aux XVI ${ }^{\mathrm{e}}$ et XVII ${ }^{\mathrm{e}}$ siècles dans les textes législatifs (ordonnances pénales ou de police, etc.), le suicide fut en revanche largement discuté dans les écrits des juristes de l'époque et notamment dans ceux de Benedict Carpzov dont la renommée rayonnait au-delà de la Saxe. Là aussi, des arbitrages pondérés prédominaient, le degré de culpabilité étant également lié à l'état mental de la victime (même si, pour Carpzov, un suicide constituait forcément un comportement condamnable) et aux particularités de chaque cas individuel (le péché ultime étant celui du malfaiteur décidé à se soustraire à sa juste peine en mettant fin à ses jours). La partie se termine par quelques observations sur la quantification du suicide (qui, à cause d'une documentation lacunaire, reste très difficile), sur les pratiques d'enterrement (avant $1700: 35$ enterrements déshonorants 
[Schandbegräbnisse], cinq «en silence " à l'extérieur du cimetière et 20 à l'intérieur, enfin sept obsèques honorables, p. 192 - le rassemblement de ces données dans un tableau aurait facilité la tâche au lecteur qui est ici et là un peu perdu face à différentes manières de compter les suicidés et les manières de les enterrer) et leurs justifications, sur la place du suicide dans les chroniques et aussi sur les réactions de la société moderne face à des suicidaires qu'elle avait réussi à identifier avant le passage à l'acte (assistance pastorale ou recours à l'enfermement par exemple; là aussi cependant le peu de dossiers conservés dans les archives fait obstacle à des conclusions plus générales).

La troisième partie enfin, de loin la plus importante $(330 \mathrm{p}$.$) , retrace d'abord$ l'élaboration et l'" acclimatation » de nouvelles normes au XVIII ${ }^{\mathrm{e}}$ siècle et notamment d'un décret publié en 1719 pour délimiter les compétences respectives des autorités laïques et ecclésiastiques après un suicide, sources de nombreux conflits et querelles qui même après cette date ne cessèrent guère ; à cette confusion s'ajoutaient également des interventions de la part de communautés opposées par exemple à l'enterrement d'un suicidé dans le cimetière de la paroisse (même si le cas inverse, la supplique demandant justement un tel enterrement, est lui aussi fréquent dans les sources). Entre 1700 et 1770 , A.K. a pu compter 36 enterrements déshonorants, sept obsèques « en silence » à l'extérieur du cimetière et 42 à l'intérieur, enfin 42 cas dont la modalité d'enterrement reste incertaine et 72 dépouilles de suicidés transférées aux "théâtres anatomiques » (p. 277 sq.) pour combler le manque criant de corps à disséquer pour des raisons pédagogiques (p. 293). Les pratiques et les contextes sociaux de cette anatomie naissante sont analysés ensuite, dans un chapitre extrêmement riche en informations nouvelles qu'il est impossible de détailler ici (cette richesse résulte en partie d'une source d'exception, le registre des dépouilles reçues par le théâtre anatomique de Dresde-Neustadt qui, pour la période de 1754 à 1817, recense 459 corps de suicidés, p.282, ce qui permet également d'obtenir quelques renseignements détaillés sur le profil social de ces derniers, p. 343-366). Parallèlement à l'avènement d'une pratique anatomique systématique, le XVIII e siècle connut d'autres évènements importants, notamment un règlement publié en 1773 concernant le sauvetage des personnes accidentées ou suicidaires. A.K. analyse non seulement les motivations (économiques et médicales) de ce texte normatif, mais également son impact sur les comportements individuels et peut ainsi constater que, même si dans les intentions des autorités un suicidaire s'assimilait désormais à la victime d'un accident, le rapport concret des sujets au suicide restait, à la fin du XVIII ${ }^{\mathrm{e}}$ siècle, équivoque et toujours aussi marqué par des traditions anciennes. Enfin, le dernier chapitre étudie la discussion et l'application d'un mandat publié en 1779 afin de régler de manière uniforme les procédures d'enterrement des suicidés et qui se distingue, par rapport à d'autres territoires, par des dispositions particulièrement sévères en la matière. Ce faisant, l'auteur aborde à son tour la question de la prétendue « décriminalisation » du suicide dans la deuxième moitié du XVIII siècle, concept cher à la recherche pour intégrer le suicide dans une histoire plus générale de la modernisation et de l'humanisation des rapports humains dans le sillage des Lumières. Les résultats saxons indiquent cependant une toute autre évolution: ici, les objectifs de prévention et de dissuasion (p.506) prévalaient largement sur tout sentiment de clémence ou de compassion. Même si les obsèques « en silence " constituent, autour de 1800, le cas habituel, 19 enterrements décrits explicitement comme « déshonorants » (schändlich) sont attestés par les sources pour la période de 1780 à 1815 (p. 519). Autrement dit, le suicide inspira toujours l'horreur à de 
larges pans de la société et resta considéré pour beaucoup comme un crime - un constat qui est renforcé par le jugement désapprobateur et donc criminalisant de l'acte suicidaire qui présidait au fréquent transfert des dépouilles de suicidés vers les salles de dissection anatomique.

De manière tout à fait convaincante, A.K. contribue ainsi lui aussi à déconstruire les grands récits d'une historiographie qui, encore aujourd'hui, élève la philosophie des Lumières au rang de fondatrice de la société contemporaine. Mais il va plus loin, en livrant un exemple éloquent de la non-linéarité de l'histoire : pendant toute la (longue) période étudiée, le traitement des suicidés (notamment leur enterrement) n'a présenté aucune homogénéité mais a varié constamment au cas par cas et en fonction de l'état mental constaté de la victime et de sa conduite avant la mort (ainsi que, dans une moindre mesure, de son statut social). Les décisions concrètes ne se mesurèrent donc pas à l'aune des seules normes (publiées de toute façon très tard) mais furent à tout moment l'objet de négociations complexes entre tribunaux, administrations, instances ecclésiastiques et entourages des suicidés. Le seul changement manifeste (en dehors de nombreuses modifications des compétences administratives et d'une importance grandissante des discours plaçant le suicide au centre de considérations économiques) porte sur une progression considérable des obsèques « en silence » vers la fin du XVIII siècle, évolution qu'il faudrait moins imputer, selon l'auteur, à une transformation des mentalités (qui auraient réagi avec davantage de compassion aux tentatives suicidaires) qu'à, d'une part, des enquêtes renforcées de la part des administrations auxquelles le mandat de 1779 imposait désormais un cadre plus strict et des catégories plus formelles de décision et, d'autre part, un recul notable de l'influence des autorités ecclésiastiques dans ce domaine (p. 548).

À l'instar d'autres chercheurs, l'auteur insiste par conséquent sur les contingences et les ambivalences des évolutions observées qui ne se résumeraient aucunement à un développement cohérent et constant vers les lueurs de la modernité et de l'humanité. C'est ici que son approche s'avère particulièrement fructueuse: en appliquant les cadres interprétatifs de l'histoire de la justice, de la police et de la domination à un champ encore largement marqué par l'idée d'un changement profond des normes et des mœurs à la fin de l'époque moderne (alors que celui-ci n'interviendra qu'au XIX ${ }^{\mathrm{e}}$ siècle, de manière lente et incomplète : voir p. 550-554) et en mobilisant une vaste documentation, y compris manuscrite (la bibliographie à la fin de l'ouvrage témoigne du nombre important de sources consultées), l'auteur réussit magistralement à ébranler quelques certitudes et à proposer une nouvelle lecture plus complexe et plus nuancée. Celle-ci ne met pas en opposition "normes » et "pratiques du quotidien », mais les analyse dans leurs imbrications réciproques pour parvenir à une image bien moins schématique et plus proche de la réalité historique que celles proposées par les "grandes" conceptions de la "sécularisation», de la "médicalisation» ou de la « décriminalisation » du suicide au XVIII ${ }^{\mathrm{e}}$ siècle. 
INDEX

Thèmes : Kulturgeschichte

Index chronologique : Frühe Neuzeit

\section{AUTEUR}

\section{FALK BRETSCHNEIDER}

École des Hautes Études en Sciences Sociales, Paris 